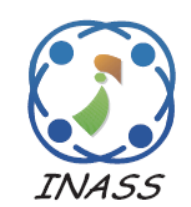

\title{
Localization of Copy-Move Forgery in Digital Images through Differential Excitation Texture Features
}

\author{
Gulivindala Suresh ${ }^{1,2 *}$ \\ Chanamallu Srinivasa Rao ${ }^{3}$ \\ ${ }^{I}$ Department of Electronics \& Communication Engineering, GMR Institute of Technology, Rajam, AP, India \\ ${ }^{2}$ Department of Electronics \& Communication Engineering, University College of Engineering-Kakinada, \\ Jawaharlal Nehru Technological University Kakinada, AP, India \\ ${ }^{3}$ Department of Electronics \& Communication Engineering, University College of Engineering-Vizianagaram, \\ Jawaharlal Nehru Technological University Kakinada, AP, India \\ * Corresponding author's Email: suresh.g@gmrit.org
}

\begin{abstract}
In this digital age, the extensive usage of digital devices and availability of open source image editing software leads to the easy manipulation of digital images. Copy-Move Forgery (CMF) is a guileless and widespread approach to hide or duplicate a certain portion of the image without leaving visual clues. Thus, it is difficult to detect the copy-move forgeries and there is a need for forensic experts to rely on an effective approach for CMF detection for forensic analysis. Hence, an efficient passive block based approach to detect and localize CMF is proposed. In the proposed method, texture features are extracted from Differential Excitation Component on the overlapping blocks of the image. Similarity measure is performed for block matching and mapping is done to identify the duplicated regions. Evaluation is performed qualitatively and quantitatively on CoMoFoD dataset; true detection rate of 0.99 and false detection rate of 0.08 has been achieved. Evaluation validates that proposed method out performs the other existing methods with regard to detection accuracy.
\end{abstract}

Keywords: Copy-move, Differential excitation, Image forgery, Texture features, Passive authentication.

\section{Introduction}

Digital images are being edited deliberately or involuntarily to make them more informative or to hide some content in the image. Vast growth of commercial and open source digital photo editing tools leads to the increase of tampered images in day-to-day life. Trustworthiness of digital image has a major role in several applications: criminal examination, journalism, forensic analysis and surveillance systems. Several techniques have been devised for Digital Image Forgery (DIF) detection and are detailed in the literature [1]. DIF detection is plausible in two approaches [2], viz., Active and Passive. The former approach involves preprocessing of genuine image by embedding an identifier before it is used. Watermarking and signature embedding methods comes under active approaches for detection and localization of image forgery. It requires pre-processing of digital data in real time and is not possible for some cameras which limit its usage. Passive approaches work without any watermark or signature. These techniques explore the statistics or features which underlay within the image during the tampering process.

Copy-Move type of forgery is one in which some snippet of an authentic image is copied and pasted at a specific location in that image itself to hide a content or duplicate a region. Copy-Move Forgery (CMF) process creates a duplicate region, which affects statistical properties of the image and these variations are explored to detect the forgery. CMF is shown in Fig. 1 and it illustrates that CMF process does not leave any visual traces and often not possible for the human eye to recognize. Therefore, detecting clues of tampering is a challenging task in image forensics. 


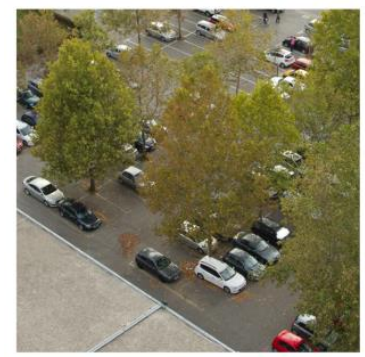

(a)

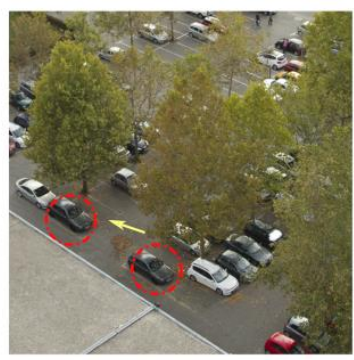

(b)
Figure. 1 Illustration of copy-move forgery: (a) original image and (b) CMF image

CMF detection techniques can be categorized as block based and key-point based methods. Keypoint based techniques rely on high entropy regions in the image to describe the features at key-points. Matching process is followed by extraction phase to detect the forged regions.

The popular and effective methods are based on features obtained from Scale Invariant Feature Transform (SIFT) [3] and Speeded Up Robust Features (SURF) [4]. A recent work on SIFT is reported [5]. Disadvantage of these techniques is they perform poorly in smooth regions. This can be addressed by block based techniques, in which features are obtained from overlapping blocks of suspicious image and these features are validated with similarity measures to detect the forgeries.

Several block based CMF detection methods are available which can handle simple CMF scenarios. However, other methods can withstand postprocessing attacks such as blurring, illumination changes, color reduction and JPEG compression. These methods work with large feature vector leads to high computational effort. To overcome this, a robust and effective method for detection of CMF through Differential Excitation Texture Features (DETF) is proposed. Main contributions of this work are: i. Texture Features (TF) are computed on DEC instead of gray image directly. DifferentialExcitation Component (DEC) captures the local salient patterns in the image in line with visual perception of human beings. This made our DETF features more distinguishable when compared with only Gray Level Co-occurrence Matrix (GLCM) TF. ii. Proposed method reveals multiple CMF and is resilient against post-processing attacks viz., brightness changes, color reduction and blurring. iii. Proposed method uses a feature vector of 24 dimension.

Remainder of the paper is organized as follows. Section 2 presents the detailed review of related works. Materials and methods of DETF feature extraction are discussed in Section 3. Proposed CMF detection method based on DETF is explained in
Section 4. Validation of proposed method with investigational results is presented in Section 5 and conclusions are given in Section 6.

\section{Literature review}

A review on block based techniques is presented in this section; the first reported work on CMF detection was developed by Fridrich et al. [6] which utilizes Discrete Cosine Transform (DCT) coefficients for feature extraction. An improved technique using Principal Component Analysis (PCA) has been developed by Popescu and Farid [7] and it has resilience against additive noise but with low detection accuracy. Guohui Li et al. [8] in his method used Discrete Wavelet Transform (DWT) to produce approximation band and Singular Value Decomposition (SVD) is employed to obtain singular values on it to form a smaller feature length when compared with [6] and [7]. A CMF detection method using blur invariant moments was introduced by Mahdian and Saic [9] and k-d tree representation was employed for block similarity analysis. This method is robust against noise, blur and contrast changes. Other moments are also explored for CMF detection and works were reported based on Pseudo-Zernike [10] and Hu [11]. These methods are robust to Additive White Gaussian Noise (AWGN), blurring and compression attacks. They are also capable to detect the CMF even if the copied portion is rotated by $20^{\circ}$ to $30^{\circ}$.

In [12], Bayram et al. developed a CMF detection method based on Fourier Mellin Transform (FMT) where in blooming filters are used instead of lexicographical sorting. A CMF detection scheme based on DCT on circular blocks was devised by Yanjun Cao et al. [13]. Their scheme used small feature vector and able to detect multiple CMFs. In [14], authors used undecimated Dyadic Discrete Wavelet Transform (DyWT) and features are obtained from approximation and detail subbands. Their method works well against compression attack. A CMF detection method [15] used Fast Walsh Hadamard Transform (FWHT) on the DWT approximation band to obtain features. The method proposed a multi-hop jump algorithm for efficient range matching. It works well against blur, noise and JPEG attacks but cannot withstand rotation and scale transforms. In order to address the problem of rotated or flipped copied portions, Leida $\mathrm{Li}$ et al. proposed a method [16] using rotation invariant Local Binary Pattern (LBP). Further, Leida et al used Polar Harmonic Transform (PHT) [17] for CMF detection. This method is resilient against affine operations, blurring, AWGN and JPEG 
compression. Histogram of Oriented Gradients (HOG) based CMF detection method [18] used a small feature vector of 4 , it is able to withstand postprocessing attacks and affine transforms to a small extent.

DCT based CMF detection method proposed by M.H. Alkawaz et al. [19] concentrated on determining the block size on the detection rate. In [20], Toqeer Mahmood et al. proposed a CMF method, explored DCT on overlapping blocks and PCA is employed for dimensionality reduction. Their method detects multiple copy-move forgeries and works well for post-processing attacks. Khizar Hayat and Tanzeela Qazi proposed a method [21] used DCT coefficients obtained from overlapping blocks of DWT approximation band for feature extraction. They developed a mask based tampering method to evaluate their algorithm. Toqeer Mahmood et al. [22] established a method using Stationary Wavelet Transform (SWT) and DCT. DCT is applied on the SWT approximation subband to obtain reduced feature vector to detect the CMF. Their method achieved robustness against attacks viz., blurring, contrast adjustments, color reduction, noise and JPEG compression.

Several CMF detection techniques are available; however, better detection accuracy with low feature vector is always substantial. For this, a robust and effective method for detection of CMF through Differential Excitation Texture Features (DETF) is proposed. Proposed method reveals multiple CMF and is resilient against post-processing attacks viz., brightness changes, color reduction and blurring. The proposed DETF method is evaluated and compared with current techniques with reference to detection rate and computational effort.

\section{Materials and methods}

In this section, DEC and novel combination of DEC with co-occurrence matrices for texture feature extraction (DETF) are presented.

\subsection{Differential excitation component}

Weber Law Descriptor [23] is a powerful local texture descriptor comprises of two components: DEC and Orientation. DEC is the ratio of change in intensity between its neighbors to a current pixel. It gives local significant patterns in the image in line with visual perception of human beings. DEC is computed as outlined in Eq.1 to 5. Firstly, change in intensity between its neighbors and the center pixel are calculated with filter $f_{00}$ and secondly, ratio of the change in intensity of the current pixel $P_{c}$ by the outputs of the two filters $f_{00}$ and $f_{01}$. Fig. 2 defines two filters $f_{00}$ and $f_{01}$.

$$
v_{s}^{00}=\sum_{i=0}^{n-1}\left(P_{i}-P_{c}\right)
$$

Where, $P_{i}(i=0,1, \ldots n-1)$ represents

$i^{\text {th }}$ neighbors of $P_{c}$ and $n$ is available neighbors.

Ratio of the intensity differences obtained from filter $f_{00}$ to the current pixel obtained from filter $f_{01}$.

$$
I_{\text {ratio }}\left(P_{c}\right)=\frac{v_{s}^{00}}{v_{s}^{01}}
$$

Employing $\arctan$ function on $I_{\text {ratio }}(\cdot)$ :

$$
\arctan \left[I_{\text {ratio }}\left(P_{c}\right)\right]
$$

Combining Eqs. (1), (2) and (3), we have

$$
\begin{aligned}
& \arctan \left[I_{\text {ratio }}\left(P_{c}\right)\right]=\arctan \left[\frac{v_{s}^{00}}{v_{s}^{01}}\right]= \\
& \arctan \left[\frac{\sum_{i=0}^{n-1}\left(P_{i}-P_{c}\right)}{P_{c}}\right]
\end{aligned}
$$

Hence, DEC of the current pixel $\zeta\left(P_{c}\right)$ is computed as:

$$
\begin{array}{r}
\zeta\left(P_{c}\right)=\arctan \left[\frac{v_{s}^{00}}{v_{s}^{01}}\right]=\arctan \left[\frac{\sum_{i=0}^{n-1}\left(P_{i}-P_{c}\right)}{P_{c}}\right] \\
\zeta\left(P_{c}\right)=\left\{\begin{array}{l}
+v e, \text { when } P_{i}>P_{c} \\
-v e, \text { when } P_{i}<P_{c}
\end{array}\right.
\end{array}
$$

Input image and its DEC scaled to 8-bit gray level are illustrated in Fig. 3.

\begin{tabular}{|l|l|l|}
\hline+1 & +1 & +1 \\
\hline+1 & -8 & +1 \\
\hline+1 & +1 & +1 \\
\hline
\end{tabular}

(a)

\begin{tabular}{|c|c|c|}
\hline 0 & 0 & 0 \\
\hline 0 & +1 & 0 \\
\hline 0 & 0 & 0 \\
\hline
\end{tabular}

(b)
Figure. 2 Filters to obtain $v_{s}^{00}$ and $v_{s}^{01}$ respectively: (a) filter $f_{00}$ and (b) filter $f_{01}$
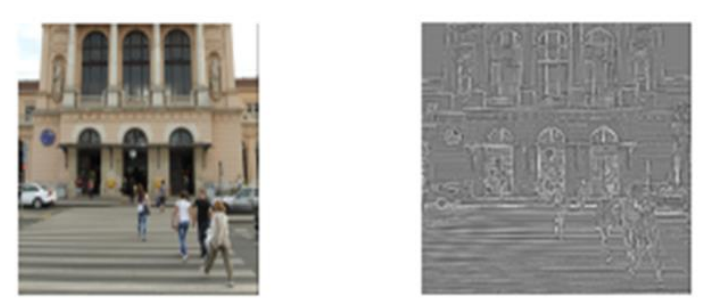

Figure. 3 RGB Image and its DEC 


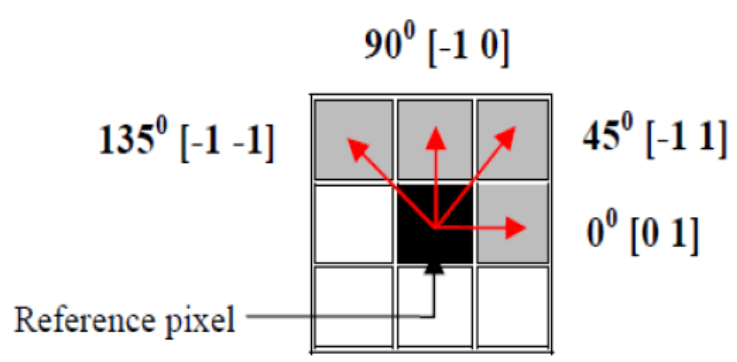

Figure. 4 DECM computation in four directions

\subsection{Differential excitation texture feature extraction}

Texture features are computed from GLCM as given by Haralick et al [24]. It is a classic approach to extract texture features for various image processing applications. Some recent applications reported based on GLCM are image retrieval [25] and image splicing [26]. In proposed method, the co-occurrence of various combinations of differential excitation components in an image segment are considered instead of gray levels to extract second order textural features from gray images.

Co-occurrence of differential excitations is calculated using two parameters, relative distance of the pixel pair $d$ and their relative orientation $\theta$. Four relative orientations for $\theta$ is considered $\{\theta=$ $\left.0^{\circ}, \theta=45^{\circ}, \theta=90^{\circ}, \theta=135^{\circ}\right\}$ to yield Differential Excitation Co-occurrence Matrices (DECM) viz., $\mathrm{DM} 4=\{\mathrm{DM}(0,1), \mathrm{DM}(-1,1), \mathrm{DM}(-$ $1,0)$ and $\mathrm{DM}(-1,-1)\}$ respectively. DECM computation is illustrated as shown in Fig. 4.

Statistical features are being computed from a normalized DECM and these are presented in Table 1. These set of 6 textural features $T F=$ $\left\{F_{1}, F_{2}, F_{3}, F_{4}, F_{5}, F_{6}\right\}$ are extracted from each DM. $T F$ extracted from $\operatorname{DM}(0,1)$ i.e $\theta=0^{\circ}$ is referred as DETF-1 $\theta$. Similarly, each orientation contributes six $T F$ and on the whole yields 24 features from all the four orientations and this feature set is referred as DETF-40. TF that are computed from GLCM in one direction (i.e $\theta=0^{\circ}$ ) is called as TF- $1 \theta$. Similarly, $T F$ from all the four orientations through GLCM is referred as TF-4 $\theta$. Feature extraction of all these four sets is illustrated in Fig. 5. The significance of the DETF- $4 \theta$ feature set is evaluated and is discussed in Section 5.

\section{Proposed method}

The vital aspect of the CMF detection techniques is to detect the duplicated regions in
Table 1. Description of texture features from DECM

\begin{tabular}{|c|c|}
\hline $\begin{array}{l}\text { Textural } \\
\text { features }\end{array}$ & Feature Description \\
\hline $\begin{array}{l}\text { Angular } \\
\text { Secondary } \\
\text { Moment }\end{array}$ & $\begin{array}{l}F_{1}=\sum_{i=1}^{n} \sum_{j=1}^{n} p^{2}(i, j) \\
F_{1} \text { measures textural uniformity }\end{array}$ \\
\hline Entropy & $\begin{array}{l}F_{2}=\sum_{i=1}^{n} \sum_{j=1}^{n} p(i, j) \log (p(i, j)) \\
F_{2} \text { defines the non-uniformity in } \\
\text { an image. Texturally non-uniform } \\
\text { image segments results in small } \\
\text { entropy. }\end{array}$ \\
\hline Contrast & $\begin{array}{l}F_{3}=\sum_{i=1}^{n} \sum_{j=1}^{n}(i-j)^{2} p(i, j) \\
\mathrm{F}_{3} \text { gives the contrast of the image. } \\
\text { Higher contrast provides clearer } \\
\text { image whereas lower contrast } \\
\text { results in a fuzzier image. }\end{array}$ \\
\hline Correlation & $\begin{array}{l}F_{4}=\frac{\sum_{i=1}^{n} \sum_{j=1}^{n}[(i, j) p(i, j)]-\mu_{1} \mu_{2}}{\sigma_{1} \sigma_{2}} \\
\text { Where, } \\
\qquad \mu_{1}=\sum_{i=1}^{n} i \sum_{j=1}^{n} p(i, j) \\
\qquad \mu_{2}=\sum_{j=1}^{n} j \sum_{i=1}^{n} p(i, j) \\
\qquad \sigma_{1}=\sum_{i=1}^{n}\left(i-\mu_{1}\right) \sum_{j=1}^{n} p(i, j) \\
\sigma_{2}=\sum_{j=1}^{n}\left(j-\mu_{2}\right) \sum_{i=1}^{n} p(i, j) \\
\text { provides gray level } \\
\text { dependencies in the image. } \\
\text { Higher correlation indicates that } \\
\text { the GLCM elements are uniform. }\end{array}$ \\
\hline $\begin{array}{l}\text { Inverse } \\
\text { Moment }\end{array}$ & $\begin{array}{l}F_{5}=\sum_{i=1}^{n} \sum_{j=1}^{n} \frac{1}{|i-j|} p(i, j) \\
\quad \text { for } i \neq j \\
\mathrm{~F}_{5} \text { measures the degree of } \\
\text { changes in the local image } \\
\text { texture. }\end{array}$ \\
\hline $\begin{array}{l}\text { Inverse } \\
\text { Difference } \\
\text { Moment }\end{array}$ & $\begin{array}{l}F_{6}=\sum_{i=1}^{n} \sum_{j=1}^{n} \frac{1}{1+(i-j)^{2}} p(i, j) \\
\mathrm{F}_{6} \text { measures image homogeneity. } \\
\text { Smaller GLCM elements results } \\
\text { in larger } \mathrm{F}_{6} .\end{array}$ \\
\hline
\end{tabular}

the given image. As well, the algorithm has to withstand affine transforms and post-processing operations, but all these are not known in advance to the forensic expert. Hence, the computational effort is more to match each potential pair of blocks, pixel by pixel. Therefore, a good set of features is very 
much essential for block matching in turn to detect forgeries.

Process flow of the proposed CMF detection method is given in Fig. 6 and outlined in the steps shown below.

Step 1: Differential excitation component for the given image is obtained.

Step 2: DEC image is divided into overlapping blocks.

Step 3: Texture features are extracted using DECM in all four orientations on DEC component.

Step 4: Block matching is performed based on the similarity measure.

Step 5: Duplicated regions are mapped on to the given image.

The proposed CMF detection method is detailed in the following points.

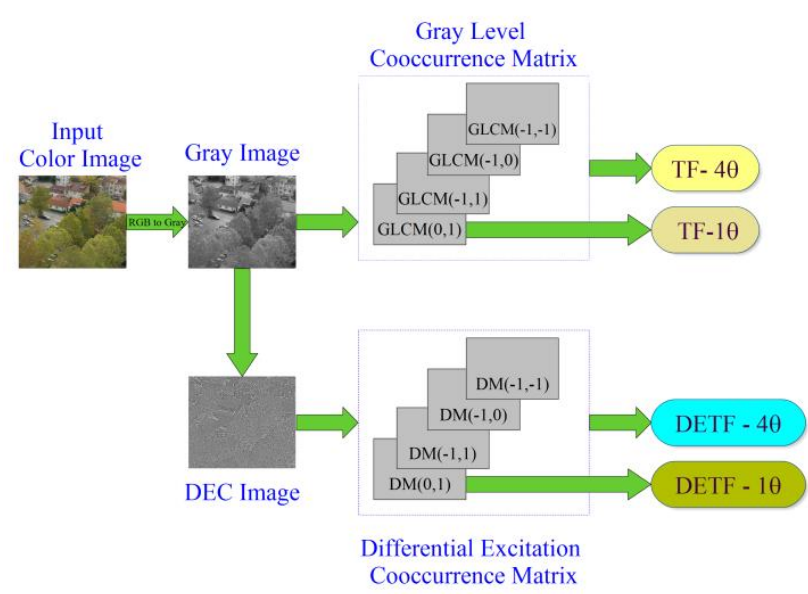

Figure. 5 Texture feature extraction from gray and DEC images

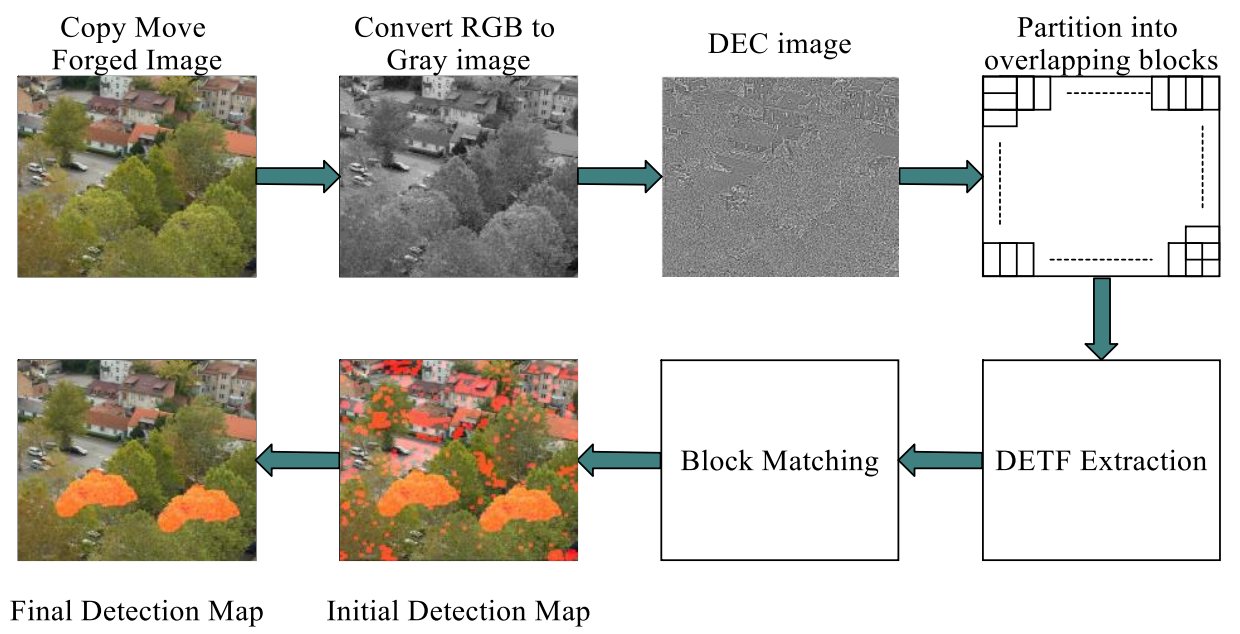

Figure. 6 Process flow of the proposed method

\subsection{Image pre-processing}

Firstly, the color image is converted into gray level image I by using:

$$
I=0.299 R+0.587 G+0.114 B
$$

Where, ' $I$ ' indicates the gray level intensity component.

Obtain DEC of the grayscale image which provides the local salient pattern in the image and is represented as $I_{D E C}$.

\subsection{Partition into overlapping blocks}

To detect CMF, the DEC image $I_{D E C}$ is partitioned into overlying blocks of fixed size ' $B$ ' to obtain texture features. Therefore, DEC image is represented with $(M-B+1) \times(N-B+1)$ overlapping blocks.

\subsection{Computation of texture features}

Six textural features are computed as described in Section 3 by applying DECM on the overlapping blocks. To make the features more distinguishable and robust, DECM is applied in four orientations viz., $\left\{\theta=0^{\circ}, \theta=45^{\circ}, \theta=90^{\circ}, \theta=135^{\circ}\right\}$ to yield DECMs DM $4=\{\operatorname{DM}(0,1), \operatorname{DM}(-1,1), \operatorname{DM}(-1,0)$ and $\mathrm{DM}(-1,-1)\}$ respectively. Each orientation contributes six textural features and on the whole a vector of 24 features vector is formed for each block. Hence, feature dimension for an image of size $M \times N$ becomes a matrix $F$ with $(M-B+$ $1) \times(N-B+1)$ rows and twenty-four columns, where twenty-four indicates the feature vector size.

\subsection{Block matching of feature set}

CMF can be detected by detecting duplicated or similar regions in the image and this is possible by 
comparing the features of all the blocks for similarity. Brute force search increases the computational complexity. In order to mitigate this issue, authors in [7] used lexicographical sorting by which the similar feature blocks are ordered. Hence, in proposed method lexicographical sorting is used on the rows of matrix $F$ so that the forged block pairs are ordered sequentially and the ordered features are represented by $S F$.

Block matching process is employed to match corresponding blocks of similar features. To achieve this, Euclidean distances of the corresponding blocks are estimated to know the similarity between them. At the same time, the feature vectors for blocks with overlapping pixels results in high similarity. For the purpose of accurate detection of CMF the blocks with physical distance greater than size of the block $B$ are considered. This is handled by a distance threshold $T_{d}$ and similarity among the corresponding blocks is validated with a similarity threshold $T_{s}$.

Block matching process takes place on the sorted matrix $S F$ as follows. The duplicated block feature set available in the $i^{\text {th }}$ row of $S F$, distances for the adjacent $r$ rows are calculated and the minimum distance is designated by $\operatorname{Dist}(i, \beta)$, given in Eq.7.

$\operatorname{Dist}(i, \beta)=\min \{\operatorname{Dist}(i, i+1), \operatorname{Dist}(i, i+$ 2), ... Dist $(i, i+r)\}$

If the obtained $\operatorname{Dist}(i, \beta)$ is less than similarity threshold $T_{S}$ then the respective blocks are considered as truly matched and their positions are stored. This procedure is carried out on the sorted matrix $S F$. The initial matched pair of blocks is saved in a set $\sigma$.

\subsection{Post-processing and final detection}

Set ' $\sigma$ ' holds all the matched block pairs and the CMF areas can be unveiled by marking the copied and forged regions. This can be achieved by marking white with black background. The initial detection result comprises of false alarms which are the false positives in a contiguous area. These are disregarded with the help of morphological operations viz., erosion and dilation. Hence, the two operators equal to block size $B$ are considered. Erosion operator when applied on the initial detection map erases the marking which is less than $B$. In contrast to this, dilation operator makes up the marking to its original size.

\section{Experimentation, results and discussion}

In this section, experimentation and validation of proposed DETF CMF detection method are elucidated. All experiments are carried out with Matlab 2017a on a computer with $2.4 \mathrm{GHz}$ processor and $8 \mathrm{~GB}$ RAM. We organized the experiments in three phases:

- Phase \#1: In this set of experiments, we concentrated on showing the significance of DETF component on detection accuracy. Experimental results with regard to the performance of proposed method are validated quantitatively and qualitatively.

- Phase \#2: In this phase of experiments, our attention is on to assess the robustness of proposed method against various post-processing attacks.

- Phase \#3: In this phase of experiments, we concentrated on comparative analysis of proposed method with existing methods reported in the literature $[3,4,7,12,16,19-22]$.

\subsection{Dataset}

Experimentation is carried on a public domain benchmark database called CoMoFoD [27], consists of images with resolution of 512 x 512 which are captured by a Canon ES camera. This set consists of forged regions being translated, rotated, scaled and a combination of these. Several post-processing attacks viz., noise addition, brightness changes, color reduction, blurring and JPEG compression are performed on the original and forged images to form a set of 10,000 images. The dataset also contains corresponding ground truths in binary and color masks.

\subsection{Performance metrics}

The efficacy of proposed method is validated by using three performance metrics at pixel level, 1. True Detection Rate (TDR), 2. False Detection Rate (FDR) and 3. Accuracy (ACC). TDR corresponds to the performance of the method in exactly detecting the CMF regions in the given image whereas FDR reflects the number of pixels that are false positives. Typical values of $T D R=1$ and $F D R=0$ indicate that the method precisely detects the CMF regions. Accuracy gives the overall metric of detection, collective value calculated from $T D R$ and FDR. It indicates the percentage of correctly located forged regions. These metrics are defined in the Eqs. (8) to (10). 


$$
\begin{gathered}
T D R=\frac{|C \cap \hat{C}|+|F \cap \hat{F}|}{|C|+|F|} \\
F D R=\frac{|\hat{C}-C|+|\hat{F}-F|}{|\hat{C}|+|\hat{F}|}
\end{gathered}
$$

Where, ' $C^{\prime}$ is the copied area and ${ }^{\prime} F^{\prime}$ is the forged area in the given forged image; ' $\widehat{C}$ ' is the detected copied area and $\widehat{C}{ }^{\prime}$ is the detected forged area resulted from the experimentation.

$$
A C C=\frac{T D R+(1-F D R)}{2} \times 100
$$

\subsection{Phase \#1: Performance of proposed method}

In this phase, experiments are carried to evaluate $T D R, F D R$ and $A C C$ of proposed method on $\mathrm{CoMoFoD}$ dataset. The images ranging from 001_F to 040_F from the dataset and their corresponding ground truths are considered for evaluation. Firstly, the significance of the proposed novel combination of texture features from DEC (DETF-49) is demonstrated. The values reported are the average values and are achieved with $T_{s}=0.01$ and $T_{d}=30$. Proposed method is evaluated for different feature sets viz., TF-1 $\theta$, DETF-1 $\theta$, TF-4 $\theta$ and DETF- $4 \theta$. TDR value for TF- $1 \theta$ is 0.79 and is improved when texture features are obtained from all the four orientations i.e TF-4 4 is 0.94 . As well, $T D R$ value for DEC i.e. DETF-1 $1 \theta$ is 0.96 and is further improved when texture features are obtained from all four orientations i.e. DETF-4 $\theta$ is 0.99 .

False positives are high when TF-1 $\theta$ is used which is 0.8006. FDR for TF-40, DETF-1 $\theta$ and DETF-4 $\theta$ are $0.31,0.22$ and 0.08 respectively. This indicates that extracting $\mathrm{TF}$ on local significant patterns obtained from DEC has improved $T D R$ and lowered FDR. This shows the significance of our novel approach of extracting DETF for CMF detection. TDR, FDR and $A C C$ for different feature sets are shown in Fig. 7, 8 and 9 respectively.

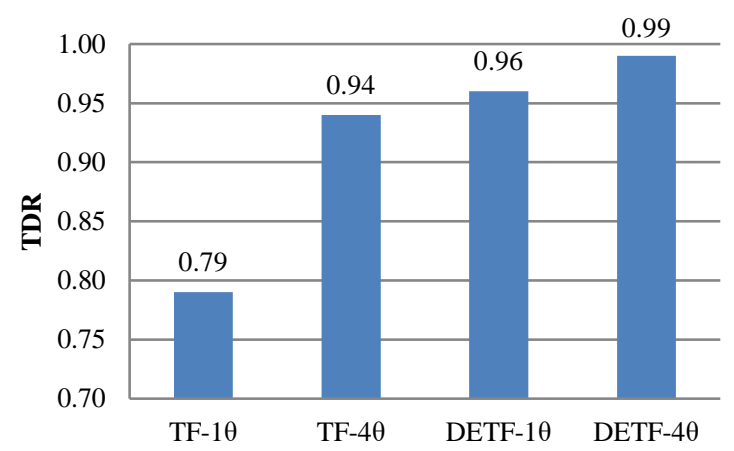

Figure. 7 TDR obtained for four feature sets

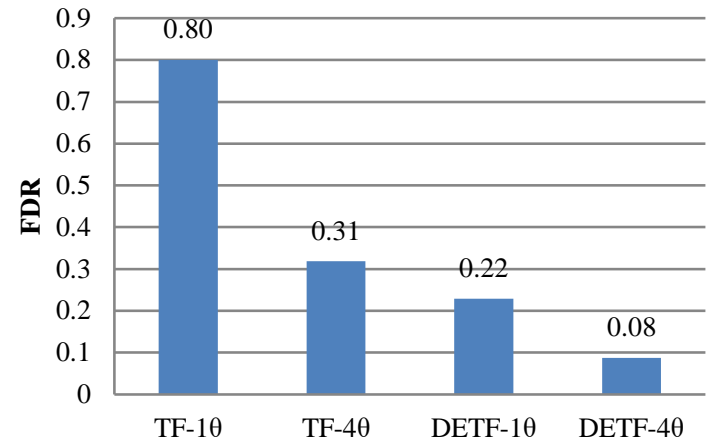

Figure. 8 FDR obtained for four feature sets

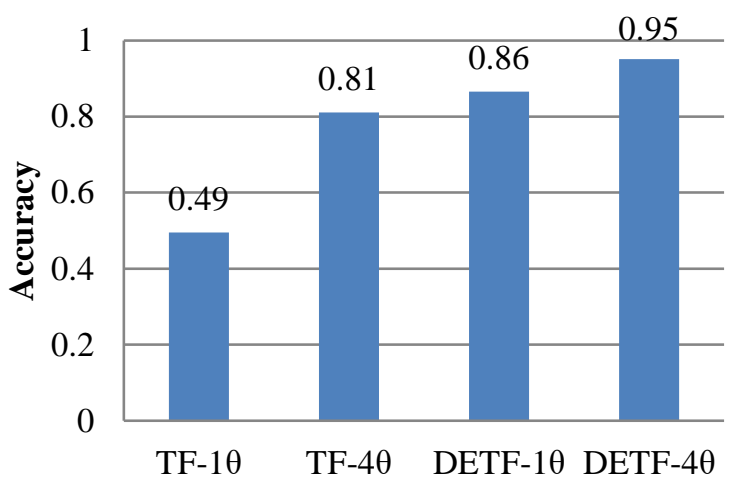

Figure. 9 Accuracy obtained for four feature sets

The performance of the proposed method with different feature sets for a sample image is presented qualitatively in the Fig. 10. The detection result obtained for the sample image using TF- $1 \theta$ feature set contains more false positives and not suitable for morphological processing.

The detection output obtained from TF-4 $\theta$ consists of less false positives and can be processed morphologically. It is demonstrated that DETF- $4 \theta$ feature set is performing better when compared with TF- $1 \theta$, DETF- $1 \theta$ and TF-4 $\theta$; hence, DETF- $4 \theta$ feature set is considered for further experimentation.

\subsection{Phase \#2: Robustness of proposed method}

In this phase, performance of proposed method is studied for different forged regions. For this, images with different forged regions such as small squares, large rectangular, large circular, irregular and multiple CMF regions are considered. Proposed method is able to detect all such cases effectively and Fig. 11 illustrates the detection results qualitatively for sample images.

Further, in this phase, robustness against different post-processing attacks is also demonstrated. 


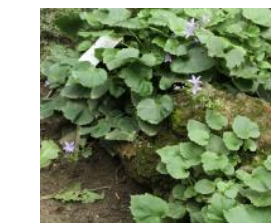

a. Original Image

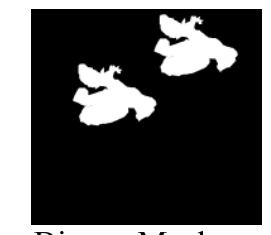

c. Binary Mask
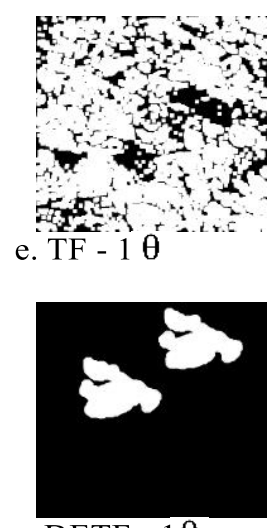

g. DETF $-1 \theta$

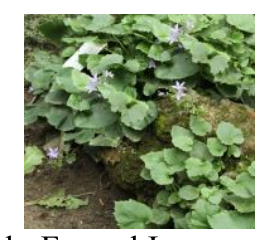

b. Forged Image

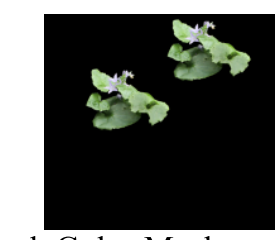

d. Color Mask
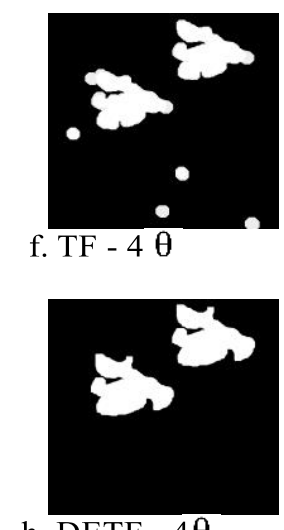

h. DETF $-4 \theta$

Figure. 10 Detection results obtained with four feature sets

\subsubsection{Brightness change attack}

To demonstrate the robustness against brightness change attack, we considered 30 images from the dataset which are processed with different brightness levels with lower and upper bounds viz., ([0.01, 0.95]), ([0.01, 0.9]) and ([0.01, 0.8]). When the image is post-processed with third level ([0.01, $0.8]$ ) then it is more imperceptible. Table 2 illustrates the detection rate of our method against brightness change. It is apparent that proposed method provides high detection rate in the presence of brightness change.

\subsubsection{Color reduction}

Robustness of the proposed method against color reduction is illustrated by considering 30 images from the dataset and quantization in color levels from 256 to 128,256 to 64 and 256 to 32. Third level of quantization 32 is visually imperceptible when compared to the other two levels as the image is represented with less number of colors. It is evident from Table 3 that proposed method provides high detection rate in the presence of color reduction.
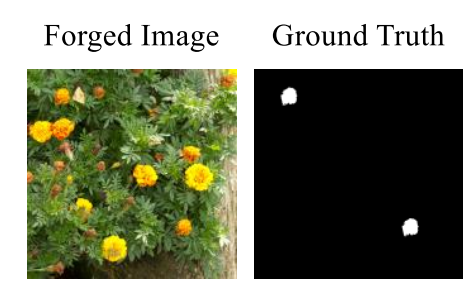

Detected Result

b
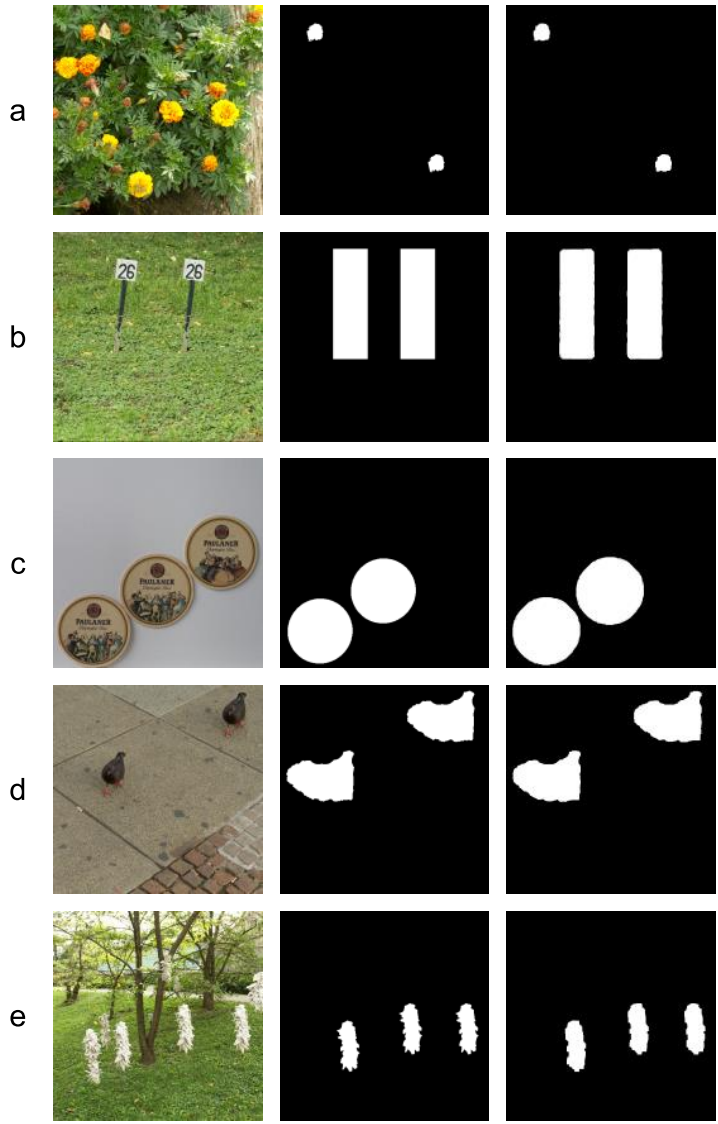

a. Small region b. Large rectangular region c. Large circular region d. Irregular region e. Multiple regions

Figure. 11 Detection results for different forged regions

Table 2. Detection rate for brightness change attack

\begin{tabular}{|c|c|c|}
\hline Brightness Change & TDR & FDR \\
\hline$[0.01,0.95]$ & 0.99 & 0.08 \\
\hline$[0.01,0.9]$ & 0.99 & 0.10 \\
\hline$[0.01,0.8]$ & 0.98 & 0.09 \\
\hline
\end{tabular}

Table 3. Detection rate for color reduction attack

\begin{tabular}{|c|c|c|}
\hline Color reduction & TDR & FDR \\
\hline 32 & 0.99 & 0.08 \\
\hline 64 & 0.99 & 0.08 \\
\hline 128 & 0.98 & 0.08 \\
\hline
\end{tabular}

\subsubsection{Image blurring}

Initially, 40 images 001_F to 040_F are processed by three different filter masks to obtain blurred images. The forged images are processed with average filters of size $3 \times 3,5 \times 5$ and $7 \times 7$. Average filter $7 \times 7$ degrades the forged image much than the other filter masks. To evaluate the efficacy in the presence of blurring, we considered 30 forged and blurred images from the dataset. Table 4 
illustrates the evaluation metrics for image blurring attack. The results shows that proposed method provides high detection rate for $3 \times 3$ filtered images but the performance degrades slightly when large filter mask such as $7 \times 7$ is used.

\subsection{Phase \#3: Comparison with existing methods}

The effectiveness of proposed method is illustrated in the previous sub sections. It shows that TDR is 0.99 and FDR is 0.08 for proposed method. Here, the comparative analysis of proposed DETF method with other state of art methods is presented. The various techniques that are considered are: PCA based [7], FMT based [12], SIFT based [3], LBP based [16], SURF based [4], DCT based [19], DCT KPCA based [20], DWT-DCT based [21], SWTDCT based [22]. The average performance comparison with brightness change, color reduction and blurring operations are illustrated in Fig. 12-17.

It is evident from Fig. 12, TDR increases when the brightness change $([0.01,0.95])$ is less and it decreases when brightness change is increased ([0.01, 0.8]) for all the methods. It is also observed that TDR is high for proposed method against this attack when compared with other methods. Similarly, false positives are high when the brightness change is more for all the methods. Fig. 13 shows the FDR and FDR of proposed method stood third relatively but out performs the other seven methods.

Table 4. Detection rate for image blurring attack

\begin{tabular}{|c|c|c|}
\hline Image blurring & TDR & FDR \\
\hline $3 \times 3$ & 0.95 & 0.05 \\
\hline $5 \times 5$ & 0.95 & 0.08 \\
\hline $7 \times 7$ & 0.93 & 0.12 \\
\hline
\end{tabular}

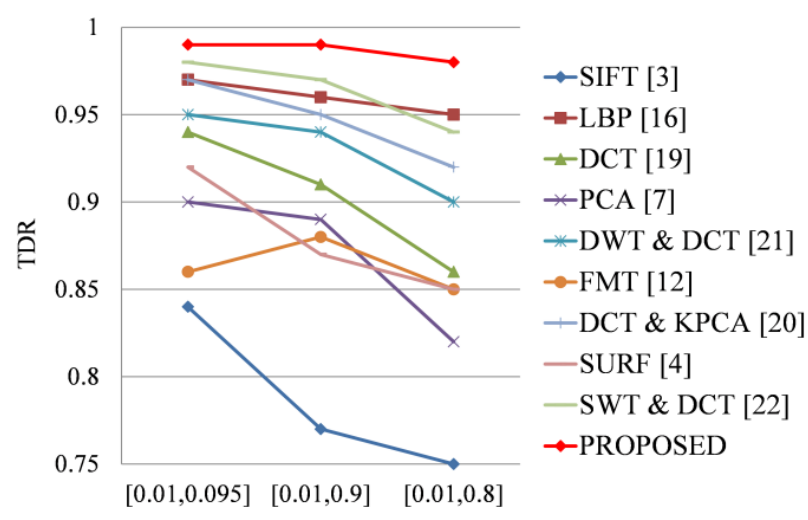

Figure. 12 TDR for brightness change

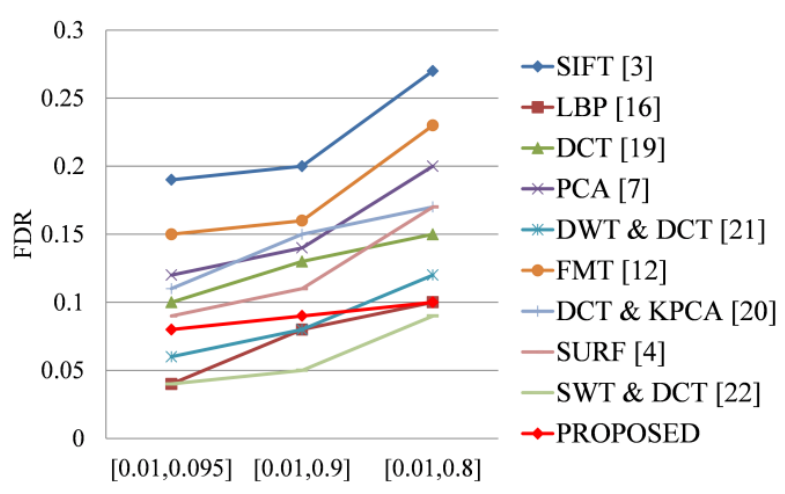

Figure. 13 FDR for brightness change

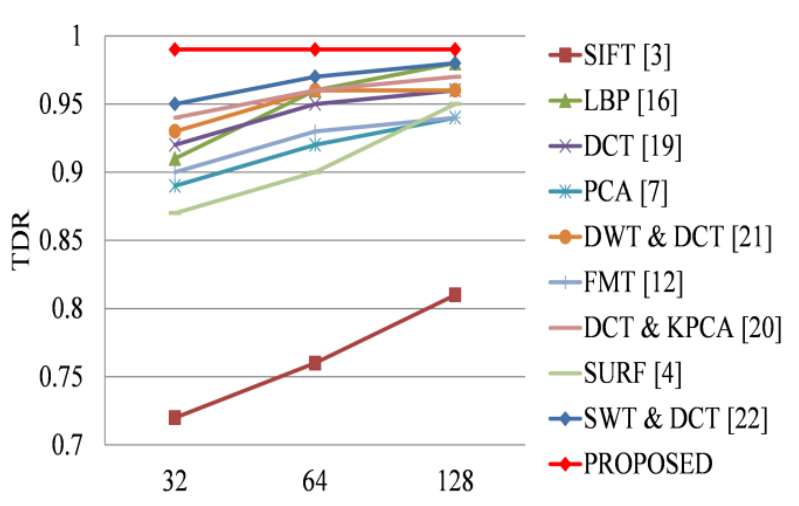

Figure. 14 TDR for color reduction

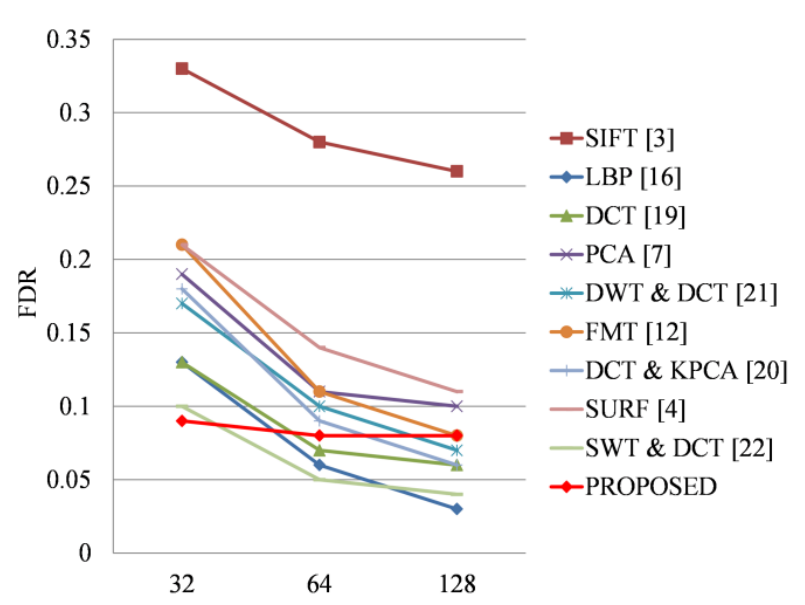

Figure. 15 FDR for color reduction

Performance of CMF detection methods in the presence of color reduction is presented in Fig. 14 and Fig. 15.

In color reduction attack, TDR decreases when the quantization level is 32 and it increases when quantization level 128 is used. The reverse is the scenario with FDR. The proposed method is superior to other methods as illustrated in Fig. 14.

The performance of CMF detection methods against blurring attack is presented in Fig. 16 \& Fig. 17. Average filter $(7 \times 7)$ attack will disturb the forged image much and this reduces the similarity 
match for features extracted. Therefore, TDR falls when average filter size increases and FDR increases. Proposed method performs well in the presence of this attack (7x7) and achieves TDR of 0.94 and FDR of 0.12 .

Also, computational complexity of proposed method is compared with other existing methods [3, $4,7,12,16,19-22]$. The computational effort is influenced by the number of overlapping blocks and feature dimension. Proposed method is evaluated on $512 \times 512$ images in block-based framework; therefore, the number of overlapping blocks is 255025 for block size of 8 . This remains the same for the block based methods as it depends on $(M-$ $B+1) \times(N-B+1)$, for an $M \times N$ size image with block size $B$. Proposed method uses a feature vector of 24 and it is smaller than the other existing CMF detection methods viz., DCT [19], SIFT [3], SURF [4], LBP [16], FMT [12] and PCA [7]. Proposed method works effectively in the presence of attacks and exhibits low computational complexity.

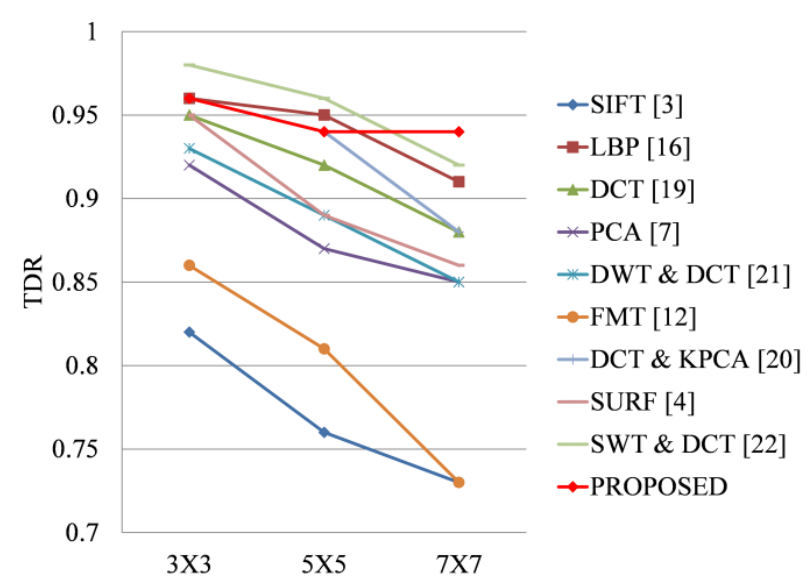

Figure. 16 TDR for blurring

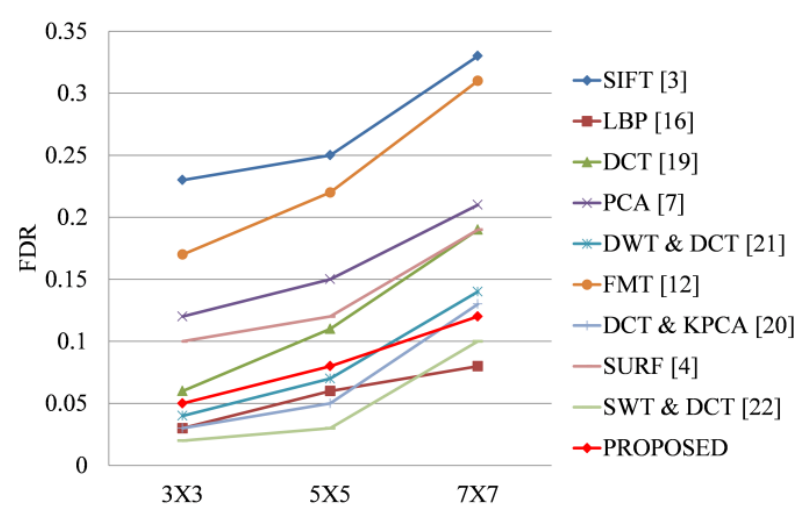

Figure. 17 FDR for blurring

\section{Conclusions}

A CMF localization method for forensic analysis in digital images is proposed in this paper. A novel CMF detection is made possible through Differential Excitation Texture Features in a block based approach. These DETF features are more distinguishable when compared to the features obtained from GLCMs. Experimentation validates that the proposed method achieved an average TDR of 0.99 and an average FDR is 0.08. Proposed DETF method is capable of detecting multiple forged regions and also is able to detect large circular, rectangular and irregular forged regions. The simulation results demonstrate that proposed method is superior to other CMF detection methods $[3,4,7$, $12,16,19-22]$ in terms of detection rate and outperforms other methods $[3,4,7,12,16,19]$ with regard to computational complexity.

The proposed method is robust against brightness change, color reduction and blurring attacks. However, proposed method does not perform well against JPEG compression attacks. In future, work can be carried to develop a robust CMF detection algorithm to withstand JPEG compression.

\section{References}

[1] M. A. Qureshi and M. Deriche, "A bibliography of pixel-based blind image forgery detection techniques", Signal Process. Image Commun., Vol. 39, pp. 46-74, 2015.

[2] H. Farid, "Image Forgery Detection A survey", IEEE Signal Process. Mag., 2009.

[3] I. Amerini, L. Ballan, S. Member, R. Caldelli, A. Del Bimbo, and G. Serra, "A SIFT-based forensic method for copy-move attack detection and transformation recovery", IEEE Trans. Inf. Forensics Secur., Vol. 6, No. 3, pp. 1099-1110, 2011.

[4] E. Silva, T. Carvalho, A. Ferreira, and A. Rocha, "Going deeper into copy-move forgery detection: Exploring image telltales via multiscale analysis and voting processes", J. Vis. Commun. Image Represent., Vol. 29, pp. 16-32, 2015.

[5] E. Mahesh, S. Maloji, and D. Rao, "A Novel Method of Scale-Invariant Feature Transform Based Image Forgery Detection", International Journal of Intelligent Engineering and Systems, Vol. 11, No. 2, pp. 132-142, 2018.

[6] J. Fridrich, D. Soukal, and J. Lukáš, "Detection of Copy-Move Forgery in Digital Images", In: Proc. of Digital Forensic Forensic Research Workshop, pp. 19-23, 2003. 
[7] A. C. Popescu and H. Farid, "Exposing digital forgeries by detecting duplicated image regions", Dept. Comput. Sci., Dartmouth Coll. Tech. Rep. TR 2004-515, No. 2000, pp. 1-11, 2004.

[8] L. Guohui, Qiong Wu, Dan Tu, Shaojie Sun; "A Sorted Neighborhood Approach For Detecting Duplicated Regions In Image Forgeries Based On DWT And SVD", In: Proc. of Multimedia and Expo, pp. 1750-1753, 2007.

[9] B. Mahdian and S. Saic, "Detection of copymove forgery using a method based on blur moment invariants", Forensic Sci. Int., Vol. 171, No. 2-3, pp. 180-189, 2007.

[10] A. H. A.-A. Khaled and W. Mahmoud, "CopyMove Forgery Detection using Zernike and Pseudo Zernike Moments", Int. Arab J. Inf. Technol., Vol. 13, No. 6, 2016.

[11] G. Liu, J. Wang, S. Lian, and Z. Wang, "A passive image authentication scheme for detecting region-duplication forgery with rotation", J. Netw. Comput. Appl., Vol. 34, No. 5, pp. 1557-1565, 2011.

[12] S. Bayram, S. Husrev Tahar, and N. Memon, "An Efficient And Robust Method For Detecting Copy-Move Forgery", In: Proc. of IEEE Acoustics, Speech and Signal Processing, pp. 1053-1056, 2009

[13] Y. Cao, T. Gao, L. Fan, and Q. Yang, "A robust detection algorithm for copy-move forgery in digital images", Forensic Sci. Int., Vol. 214, No. 1-3, pp. 33-43, 2012.

[14] G. Muhammad, M. Hussain, and G. Bebis, "Passive copy move image forgery detection using undecimated dyadic wavelet transform", Digit. Investig., Vol. 9, No. 1, pp. 49-57, 2012.

[15] Y. Bin, S. Xingming, and C. Xianyi, "An Efficient Forensic Method for Copy - Move Forgery Detection Based on DWT-FWHT", Radioengineering, Vol. 22, No. 4, pp. 1098$1105,2013$.

[16] L. Li, S. Li, H. Zhu, S.-C. Chu, J. F. Roddick, and J.-S. Pan, "An efficient scheme for detecting copy-move forged images by local binary patterns", J. Inf. Hiding Multimed. Signal Process., Vol. 4, No. 1, pp. 46-56, 2013.

[17] L. Leida, L. Shushang, Z.Hancheng, and Wu. Xiaoyue, "Detecting copy-move forgery under affine transforms for image forensics", Comput. Electr. Eng., Vol. 40, No. 6, pp. 1951-1962, 2014.

[18] J. C. Lee, C. P. Chang, and W. K. Chen, "Detection of copy-move image forgery using histogram of orientated gradients", Inf. Sci. (Ny)., Vol. 321, pp. 250-262, 2015.
[19] M. H. Alkawaz, G. Sulong, T. Saba, and A. Rehman, "Detection of copy-move image forgery based on discrete cosine transform", Neural Comput. Appl., Vol.30, pp. 183-192, 2016.

[20] T. Mahmood, T. Nawaz, A. Irtaza, R. Ashraf, M. Shah, and M. T. Mahmood, "Copy-Move Forgery Detection Technique for Forensic Analysis in Digital Images", Math. Probl. Eng., Vol. 2016, pp. 1-13, 2016.

[21] K. Hayat and T. Qazi, "Forgery detection in digital images via discrete wavelet and discrete cosine transforms", Comput. Electr. Eng., Vol. 62, pp. 448-458, 2017.

[22] T. Mahmood, Z. Mehmood, M. Shah, and T. Saba, "A robust technique for copy-move forgery detection and localization in digital images via stationary wavelet and discrete cosine transform", J. Vis. Commun. Image Represent., Vol. 53, No. 3, pp. 202-214, 2018.

[23] J. Chen, S. Shiguang, H. Chu, Z. Guoying, M. Pietikäinen, X. Chen, and G. Wen, "WLD : A Robust Local Image Descriptor", IEEE Trans. Pattern Anal. Mach. Intell., Vol. 32, No. 9, pp. 1-16, 2009.

[24] R. M. Haralick, K. Shanmugam, and I. Dinstein, "Textural Features for Image Classification", IEEE Trans. Syst. Man. Cybern., Vol. SMC-3, No. 6, pp. 610-621, 1973.

[25] M. K. Alsmadi, "Query-sensitive similarity measure for content-based image retrieval using meta-heuristic algorithm", J. King Saud Univ. Comput. Inf. Sci., Vol. 30, pp. 373-381, 2017.

[26] X. Shen, Z. Shi, and H. Chen, "Splicing image forgery detection using textural features based on the grey level co-occurrence matrices", IET Image Process., Vol. 11, No. 1, pp. 44-53, 2017.

[27] D. Tralic, I. Zupancic, S. Grgic, and M. Grgic, "CoMoFoD - New Database for Copy-Move Forgery Detection", In: Proc.of the 55th Int. Symp. ELMAR-2013, pp. 49-54, 2013. 